\title{
A Suspended Laser Interferometer for Determining the Newtonian Constant of Gravitation
}

\author{
Harold V. Parks, James E. Faller, and Douglas S. Robertson
}

\begin{abstract}
Progress is reported on an experiment to measure the Newtonian constant of gravitation $G$ with a suspended FabryPerot laser interferometer. With this technique, we measure the deflection of simple pendulums due to the gravitational attraction of tungsten masses. A result for $G$ is expected with a relative uncertainty of $3 \times 10^{-5}$.
\end{abstract}

Index Terms-Fabry-Perot interferometer, gravity measurement, Newtonian constant.

\section{INTRODUCTION}

$\mathbf{T}$ HE Newtonian constant of gravitation $G$ is one of the least well known of the fundamental constants. The accepted value has, for many years, been assigned a relative uncertainty of $1.28 \times 10^{-4}[1]$. However, several recent precision measurements disagree with this value by over $10 \sigma$ (one is high [2] and the other is low [3]), and others disagree by $2 \sigma$ [4], [5] (again in opposite directions). In light of these disagreements, the relative uncertainty in the CODATA value has recently been increased to $1.5 \times 10^{-3}$. This situation highlights the need for new measurements using a variety of different methods. While most measurements of $G$ use torsion pendulums or balances, we sense the deflection of simple pendulums. This results in a conceptually very simple system. Rather than relying on the torsion of a wire, which is not well understood to the accuracy required, we measure directly the ratio of the attraction of a laboratory source mass and the attraction of the earth. This approach has been used once before by a group at Wuppertal, Germany, using microwaves to measure the pendulum deflections [2]. The use of optical interferometry in our experiment gives greater sensitivity and permits a much more compact and manageable apparatus. This allows the source mass design to be optimized, which is a point that is critical to achieving the full potential of this method. In the following sections, we give an overview of our apparatus and then discuss the source mass geometry followed by the laser measurement technique and system performance.

\section{EXPERIMENTAL APPARATUS AND METHOD}

\section{A. Overview}

In this experiment, two simple pendulums are hung inside a vacuum chamber from a common support, as is shown schematically in Fig. 1. Since we are only interested in the separation

Manuscript received May 14, 2000; revised October 27, 2000.

H. V. Parks and J. E. Faller are with the Joint Institute for Laboratory Astrophysics, National Institute of Standards and Technology, University of Colorado, Boulder, CO 80309-0440 USA.

D. S. Robertson is with the National Geodetic Survey, NOS, NOAA, CIRES, and the University of Colorado, Boulder, CO 80309-0216 USA.

Publisher Item Identifier S 0018-9456(01)02667-5.

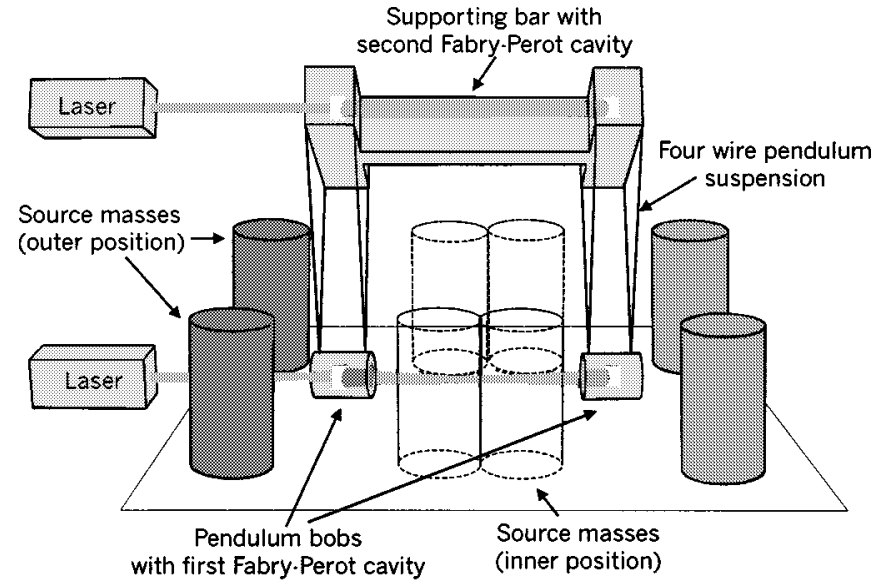

Fig. 1. Schematic of the apparatus. Two pendulums hang from a common support. Tungsten source masses in the form of four vertical cylinders pull the pendulum bobs apart in the outward position (solid lines) and together when they are moved to the inward position (dashed lines). A vacuum chamber, which is not shown here, encloses the pendulums and supporting bar but not the source masses. Lasers measure the change in the separation of the two pendulum bobs as well as any thermal expansion in the supporting bar.

of the two pendulum bobs, the effects of noise from seismic vibration and distant gravitational sources are greatly reduced because the effects will be common to both bobs. Large tungsten source masses are first placed between the two test masses (the pendulum bobs), and the resulting gravitational attraction pulls the two test masses together. The source masses are then separated and moved beyond the ends of the pendulum system, causing the test masses to be pulled outward.

The plate supporting the source masses rests on three feet. Since the center of mass of the source mass system never moves, there is no induced tilt in the floor, which could cause an experimental error. The change in test mass separation is monitored with a Fabry-Perot laser interferometer. A second laser interferometer spanning the pendulum supports compensates for any thermally induced change in the separation between the pendulum support points; the actual measurement is made by beating the two lasers together. This method automatically subtracts any thermal component from the motion of the pendulum bobs.

The pendulums have a length of $70 \mathrm{~cm}$ and a separation of 30 $\mathrm{cm}$. Since each pendulum bob contains an interferometer mirror, which must remain aligned, each bob is supported by four wires: two wires that meet in a " $v$ " at either end of the bob (see Fig. 1). This assures that when the bobs move, they undergo parallel translation with no twist. Each of the source mass cylinders has a mass of $119 \mathrm{~kg}$, a height of $30 \mathrm{~cm}$, and a diameter of $16 \mathrm{~cm}$. The source masses are located $16 \mathrm{~cm}$ to each side of the Fabry-Perot 
cavity axis. Each of the source mass cylinders is made up of a stack of three smaller cylinders. The distance between the center of the pendulum bobs and the centers of each of the two nearest source mass cylinders is $23 \mathrm{~cm}$ when the source masses are in the inner position and $34 \mathrm{~cm}$ when the source masses are in the outer position. The gravitational effect of moving the source masses from the inward to the outward position will change the pendulum bob separation by about $120 \mathrm{~nm}$.

\section{B. Source Mass Geometry}

Since the accuracy of our method is likely to be ultimately limited by imperfections in the geometry and density of the source masses, care is taken in the experimental design. It is well known that a ring-shaped source mass can reduce sensitivity to source mass-test mass positioning errors. Consider the gravitational field along the axis of the ring. There is a point on either side of the ring where the gravitational pull toward the ring is maximized. Further, it turns out that gradient of all three components of the gravitational field is zero at this point. The effect of this is dramatic. In order to determine $G$ to a few parts in $10^{5}$ by placing a single spherical source mass $20 \mathrm{~cm}$ from a test mass, the distance between the two masses would have to be measured to an accuracy on the order of $10 \mu \mathrm{m}$. However, if a ring were used, a source mass-test mass positioning error of almost $1 \mathrm{~mm}$ could be tolerated.

The system is, however, still sensitive to the radius of the ring. In our case, the plane of the ring would have to be vertical, causing the ring to sag out of its perfectly circular shape, complicating the situation still further. This problem can be avoided by a modification to the source mass design. We replace the ring by two cylinders located symmetrically on either side of the laser beam, as shown in the left (or right half) of Fig. 1. With this geometry, the gradient of the components gravitational field directed along the laser beam ( $z-)$ axis is still zero, and now, the system is sensitive only to the separation of the centers of mass of the two source mass cylinders. The apparatus is quite insensitive to tilts and displacements of these cylinders, as long as the center of mass separation is maintained. The gradients of the components of the gravitational field perpendicular to the $z$-axis are no longer zero, but the instrument is not very sensitive to these components, so little is lost. Source mass-test mass positioning errors of nearly $1 \mathrm{~mm}$ can still be tolerated. Note that in the actual experiment, four cylinders are used so that each of the pendulum bob test masses can be pulled in opposite directions. These four cylinders are placed so that gradient of the gravitational field along the $z$-axis is zero at the location of each pendulum bob.

The above argument assumes that the source masses have a constant density. In any real experiment, the source masses will have some density variation, which may cause errors in the final determination of $G$. Once the experiment is performed, it can be repeated with each of the source mass cylinders rotated around its symmetry axis to test for density variations in the source masses. In addition, each cylinder is made up of a stack of three shorter cylinders, which can be shuffled. This leaves the possibility of an undetectable common radial density variation in the source masses. We have found, however, that optimizing the distance between the source masses and the test masses can reduce this error.

\section{Laser Interferometer}

The separation of the two test masses is monitored with a laser interferometer. Mirrors embedded in each of the test masses form a Fabry-Perot cavity, and a He-Ne laser is locked to this cavity. This laser system was constructed at the Joint Institute for Laboratory Astrophysics (JILA) and is quite economical. The He-Ne laser tube is short enough so that only one longitudinal mode (of a particular polarization) is produced. The frequency of this mode is controlled with two stages. First, a heater is used to servo the length of the laser tube. However, this method is too slow to track any swinging or vibration of the pendulum; therefore, a double-passed acousto-optic modulator is used when a fast response is needed. Although the acousto-optic modulator gives a much faster response than the thermal method, it has a much smaller tuning range: $100 \mathrm{MHz}$ for the acousto-optic modulator compared with 1 $\mathrm{GHz}$ for thermal tuning. The laser beam is then sent through an electro-optic modulator to provide the phase modulation necessary for a Pound-Drever lock. When the source masses move from the inner to the outer position (which is indicated in Fig. 1), the separation of the test masses will change by approximately $120 \mathrm{~nm}$, resulting in a change of $90 \mathrm{MHz}$ in the frequency of the laser. A support point interferometer measures the separation between the pendulum support points. Another laser is locked to this cavity, and the displacement measurements are made by beating these two lasers together. In this way, much of the error due to thermal drift in the length of the supporting bar is cancelled out.

\section{Performance}

Although the apparatus is not complete, we have locked the laser to the pendulum cavity and observed a gravitationally induced change in test mass separation. The laser system itself is capable of measuring the expected $120 \mathrm{~nm}$ cavity length change to an accuracy of better than 1 part in $10^{5}$ with only a few minutes of averaging. However, the largest contribution to the signal noise is not the laser system but the pendulums swinging at the fundamental resonance driven by seismic motion. By positioning magnets underneath the pendulum bobs, this noise can be reduced by eddy current damping. In a jury-rigged test setup, we were able to reduce the amplitude of the differential mode pendulum motion to several nanometers. We are now completing both a much more rigid support for the pendulums and a mechanism to allow the pendulum lengths to be accurately matched. In addition, the apparatus is currently located at JILA on a busy university campus. Moving it to a less-populated area nearby will reduce the seismic noise by an order of magnitude.

The influence of the damping magnets on pendulums has not yet been thoroughly studied. We move the magnets by an amount equal to the predicted pendulum displacement so that the relative position of the magnet and the pendulum bob does not change, eliminating any errors due to paramagnetic forces or contamination of the bob by ferromagnetic material. The eddy current damping will have to be removed to measure the period 
of the pendulums and thus calibrate the pendulum displacement with a force. This can be done by moving the magnets away from the bobs or by moving the magnets back and forth at the pendulum frequency.

\section{CONCLUSION}

Our experiment to measure the Newtonian constant of gravitation is currently under development. The method is different from the majority of other experiments to determine $G$ in that we use simple pendulums instead of torsion pendulums or balances. Thus, we measure directly the ratio of the attraction of the laboratory source masses to the earth's gravity. With optical interferometric techniques, it is straightforward to measure even the small deflections of the simple pendulums. Care is taken to minimize errors due to imperfections in the source masses. It will be possible to take measurements quickly with this apparatus so that the experiment can be repeated with the source masses in a different configuration, allowing errors due to small density variations inside the source mass to be detected. We hope to be able to determine $G$ with a relative uncertainty of $3 \times 10^{-5}$ with this apparatus.

\section{REFERENCES}

[1] G. G. Luther and W. R. Towler, "Redetermination of the Newtonian gravitational constant," Phys. Rev. Lett., vol. 48, pp. 121-123, 1982.

[2] W. Michaelis, H. Haars, and R. Augustin, "A new precise determination of Newton's gravitational constant," Metrologia, vol. 32, pp. 267-276, 1995.

[3] M. P. Fitzgerald and T. R. Armstrong, "Newton's gravitational constant with uncertainty of less than 100 ppm," IEEE Trans. Instrum. Meas., vol. 44, pp. 494-497, Apr. 1995.

[4] A. S. Schumacher et al., "Determination of the gravitational constant $G$ using a Fabry-Perot pendulum resonator," in Tech. Dig. CPEM, Washington, DC, July 1998, pp. 144-145.

[5] J. Schurr, F. Nolting, and W. Kundig, "Gravitational constant measured by mean of a beam balance," Phys. Rev. Lett., vol. 80, pp. 1142-1145, 1998. 